Fetal Diagnosis and Therapy
Fetal Diagn Ther 2017;41:251-257

DOI: $10.1159 / 000448753$
Received: March 23, 2016

Accepted after revision: July 14, 2016

Published online: October 12, 2016

\title{
Fetal and Neonatal Alloimmune Thrombocytopenia: Management and Outcome of a Large International Retrospective Cohort
}

\author{
Marije M. Kamphuis ${ }^{a}$ Heidi Tiller, d E.S. van den Akker ${ }^{b}$ Magnus Westgren ${ }^{e}$ \\ Eleonor Tiblad ${ }^{\mathrm{e}}$ Dick Oepkes $^{\mathrm{a}}$ \\ a Department of Obstetrics, Leiden University Medical Centre, Leiden, and ${ }^{\mathrm{b}}$ Onze Lieve Vrouwe Gasthuis, \\ Amsterdam, The Netherlands; ${ }^{C}$ Immunology Research Group, Department of Medical Biology, UiT - The Arctic \\ University of Norway, and ${ }^{d}$ Department of Obstetrics and Gynecology, University Hospital of Norway, Troms $\varnothing$,

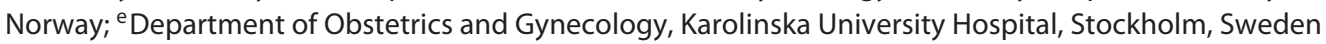

\section{Key Words}

Fetal and neonatal alloimmune thrombocytopenia - Large cohort · Antenatal treatment · Intracranial haemorrhage · Fetal therapy

\begin{abstract}
Objective: To evaluate the management and outcome of a large international cohort of cases of pregnancies complicated by fetal and neonatal alloimmune thrombocytopenia (FNAIT). Methods: This was an observational prospective and retrospective cohort study of all cases of FNAIT entered into the international multicentre No IntraCranial Haemorrhage $(\mathrm{NOICH})$ registry during the period of 2001-2010. We evaluated human platelet antigen (HPA) specificity, the antenatal and postnatal interventions performed, and clinical outcome. Results: A total of 615 pregnancies complicated by FNAIT from 10 countries were included. Anti-HPA-1a was the most commonly implicated antibody. Antenatal treatment was administered in 273 pregnancies (44\%), varying from intrauterine platelet transfusion to maternal administration of immunoglobulins, steroids, or a combination of those. Intracranial haemorrhage was diagnosed in 23 fetus-
\end{abstract}

\begin{tabular}{ll}
\hline KARGER & $\begin{array}{l}\text { ( ) 2016 The Author(s) } \\
\text { Published by S. Karger AG, Basel }\end{array}$ \\
$\begin{array}{l}\text { E-Mail karger@karger.com } \\
\text { www.karger.com/fdt }\end{array}$ & $\begin{array}{l}\text { Karger } \\
\text { This article is licensed under the Creative Commons Attribution- } \\
\text { NonCommercial-NoDerivatives 4.0 International License (CC BY- } \\
\text { NC-ND) (http://www.karger.com/Services/OpenAccessLicense). } \\
\text { Usage and distribution for commercial purposes as well as any dis- } \\
\text { tribution of modified material requires written permission. }\end{array}$
\end{tabular}

es or neonates (3.7\%). Overall perinatal mortality was $1.14 \%$ $(n=7)$. Conclusion: This study presents the largest cohort of cases of FNAIT published. Our data show that antenatal treatment for FNAIT results in favourable perinatal outcome. Over time, in most centres, treatment for FNAIT changed from an invasive to a complete non-invasive procedure.

\author{
(c) 2016 The Author(s) \\ Published by S. Karger AG, Basel
}

\section{Introduction}

Fetal and neonatal alloimmune thrombocytopenia (FNAIT) is caused by an immunological process in which the mother produces an antibody-mediated response against a platelet-specific antigen [human platelet antigen (HPA)] that she herself lacks but that is present on the fetal platelets, inherited from the father [1]. The mother's antibodies, of the IgG (immunoglobulin G) type, can cross the placenta and bind to fetal platelets. The antibody-coated platelets are subsequently removed from the fetal circulation by the reticuloendothelial system, which results in fetal thrombocytopenia. These same antibodies

Dick Oepkes

Department of Obstetrics K6-35, Leiden University Medical Centre PO Box 9600

NL-2300 RC Leiden (The Netherlands)

E-Mail d.oepkes@lumc.nl 
Fig. 1. Participating centres. LUMC = Leiden University Medical Centre; $\mathrm{UHNN}=$ University Hospital North Norway; UHSW = University Hospital South Norway.

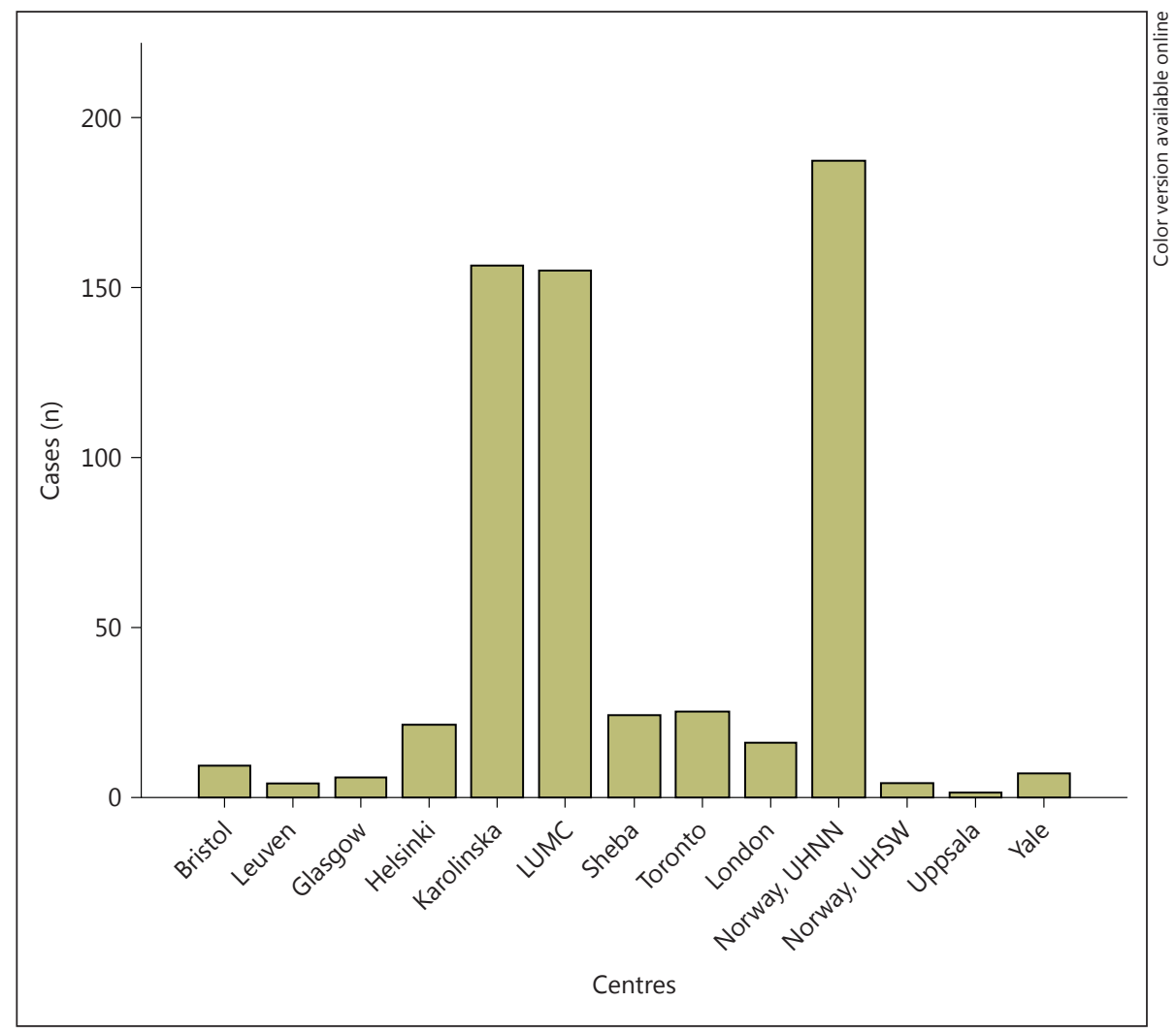

also may inhibit platelet production [2]. The proportion of individuals belonging to a particular platelet antigen type varies according to ethnicity. The immunodominant antigen in Caucasian individuals is HPA- $1 \mathrm{a}$, which is responsible for $85 \%$ of FNAIT cases, followed by HPA-5b in $10 \%$ [3]. Two percent of Caucasians are HPA-1 a negative. The reported incidence of FNAIT ranges from 1:350 to $1: 1,000[4]$. The most severe complication is intracranial haemorrhage (ICH), leading to lifelong handicap or even death. The clinical outcome is often more severe than that of neonatal ICH from other causes [3-6]. The majority of ICH bleedings seem to occur by the end of the 2nd trimester [7].

Without routinely screening for HPA antibodies, the disease is nowadays diagnosed after the birth of the first affected child. Subsequently, antenatal treatment can only be offered in following pregnancies to avoid recurrence of severe FNAIT.

The aim of this study was to analyse the management and outcome of the largest international cohort of FNAIT cases to date, with emphasis on the different treatment modalities.

\section{Material and Methods}

\section{Data Collection}

The No IntraCranial Haemorrhage $(\mathrm{NOICH})$ registry database (http://www.NOICH.org) was initially set up in 2001 for the NOICH study, an international multicentre randomized controlled trial comparing 0.5 with $1.0 \mathrm{~g}$ intravenous immunoglobulin (IVIG) for the prevention of bleeding in fetuses and neonates at risk of FNAIT. This study was prematurely ended in 2008 due to lack of inclusion [8]. The registry was kept open for fetal treatment centres worldwide to enter data on pregnancies complicated by FNAIT. Data were entered both retrospectively and prospectively by 13 tertiary referral centres from 10 different countries around the world (fig. 1). An observational cohort study of these data was performed.

\section{Cases}

A case was defined as affected by FNAIT if incompatibility between the maternal and the paternal/fetal HPA type was confirmed and maternal anti-HPA antibodies were detected. Initially, cases were entered into the database for inclusion in the NOICH randomized trial. All non-randomized and non-eligible cases from the participating centres were included as well. After having prematurely ended the trial, the contributing centres kept collecting cases. These were mainly referred patients known to be at risk of FNAIT because of a previous affected child. Some cases originated from a previous Norwegian screening study of FNAIT [9]. Fetuses and neonates with major congenital or chromosomal abnormalities were excluded.
Kamphuis/Tiller/van den Akker/ Westgren/Tiblad/Oepkes 
Table 1. HPA specifics

\begin{tabular}{lclc}
\hline HPA type & Cases, $\mathrm{n}(\%)$ & Mean PC $\times 10^{9} / 1$ & $\mathrm{ICH}, \mathrm{n}$ \\
\hline HPA-1a & $544(88)$ & 105 & 19 \\
HPA-5b & $23(3.6)$ & 136 & 2 \\
HPA-3a & $7(1.1)$ & 147 & \\
HPA-5a & $4(0.6)$ & 184 & 2 \\
HPA-15a & $5(0.8)$ & 200 & \\
HPA-1a + -5b & $18(3)$ & 94 & \\
HPA-1a + other & $5(0.8)$ & & \\
Negative & $2(0.03)$ & & \\
Unknown & $7(1.1)$ & & \\
Total & 615 & \\
\hline \multicolumn{2}{c}{ PC = Platelet count. } \\
\hline
\end{tabular}

Outcome

The primary outcome variables were HPA specificity, maternal/neonatal demographic characteristics, and clinical outcome. Cases affected by ICH were not extensively described; we refer to our previously published paper for the characteristics of the $\mathrm{ICH}$ group [7]. In that study, both older and younger siblings with $\mathrm{ICH}$ were also identified and included, resulting in a cohort of 43 cases. In the current study, we focus only on the $\mathrm{ICH}$ cases that were originally included in the $\mathrm{NOICH}$ registry database, explaining the lower number $(\mathrm{n}=23)$ described in this paper.

Secondary outcome variables were antenatal and postnatal interventions performed in pregnancies complicated by FNAIT. Outcomes of pregnant women with a previous child with $\mathrm{ICH}$, considered as at high risk, are described separately.

To set up the database, approval was given by the Leiden University Medical Centre's Medical Ethics Committee (MEC PP04.203) and by each centre's respective Institutional Review Board.

\section{Statistical Analysis}

Categorical data are summarized as actual numbers and percentages. Continuous data are presented as medians or means with ranges. The analysis was done using the independent-samples $t$ test. A p value of 0.05 was considered statistically significant. Data analysis was generated using SPSS software (version 20; SPSS Inc., Chicago, Ill., USA).

\section{Results}

The NOICH registry database contained data on 615 pregnancies complicated by FNAIT; part of these were registered prospectively: 23 for inclusion into the $\mathrm{NOICH}$ trial [8] and 177 as a result of the screening study in Norway [9]. Figure 1 shows the contributions of the participating centres, a major part of which are situated in Nor-
Table 2. Maternal and neonatal demographics

\begin{tabular}{|c|c|}
\hline \multicolumn{2}{|l|}{ Pregnancy type } \\
\hline Singleton & $599(97)$ \\
\hline Multiple & $16(3)$ \\
\hline \multicolumn{2}{|l|}{ Parity $^{\mathrm{a}}$} \\
\hline Primiparous & $100(19)$ \\
\hline Multiparous & $499(83)$ \\
\hline \multicolumn{2}{|l|}{ History } \\
\hline FNAIT $^{\mathrm{b}}$ & $343(56)$ \\
\hline IUFD & $23(7)$ \\
\hline $\mathrm{ICH}$ & $50(15)$ \\
\hline \multicolumn{2}{|l|}{ Mode of delivery ${ }^{\mathrm{c}}$} \\
\hline Vaginal & $240(39)$ \\
\hline Instrumental & $4(0.7)$ \\
\hline Elective caesarean & $289(50)$ \\
\hline Emergency caesarean & $46(7.5)$ \\
\hline \multicolumn{2}{|l|}{ Birth weight ${ }^{\mathrm{d}}, \mathrm{g}$} \\
\hline Mean (range) & $2,985(400-4,775)$ \\
\hline \multicolumn{2}{|l|}{ Gestational age, weeks } \\
\hline Mean (range) & $37(24-42)$ \\
\hline \multicolumn{2}{|l|}{$\operatorname{Sex}^{\mathrm{e}}$} \\
\hline Boys & 229 (55) \\
\hline Girls & $188(45)$ \\
\hline \multicolumn{2}{|l|}{ Platelet count at birth ${ }^{\mathrm{f}}$} \\
\hline Mean (range) $\times 10^{9} / 1$ & $108(1-404)$ \\
\hline$<30 \times 10^{9} / 1$ & $191(31)$ \\
\hline$<50 \times 10^{9} / 1$ & $235(43)$ \\
\hline $50-100 \times 10^{9} / 1$ & $78(14)$ \\
\hline $100-150 \times 10^{9} / 1$ & $59(11)$ \\
\hline$>150 \times 10^{9} / 1$ & $172(32)$ \\
\hline Petechiae & $100(17)$ \\
\hline Petechiae and PC $<30 \times 10^{9} / 1$ & $81(81)$ \\
\hline $\mathrm{ICH}$ & $23(3.7)$ \\
\hline IUFD & $4(0.7)$ \\
\hline
\end{tabular}

Values are presented as $\mathrm{n}(\%)$ unless specified otherwise. ${ }^{\mathrm{a}} 17$ cases missing. ${ }^{b} 154$ cases missing. ${ }^{c} 36$ cases missing. ${ }^{\mathrm{d}} 71$ cases missing. ${ }^{\mathrm{e}} 198$ cases missing. ${ }^{\mathrm{f}} 40$ cases missing.

way, Sweden, and the Netherlands. The HPA antibody specifics are outlined in table 1, with anti-HPA-1a being the most commonly implicated antibody ( $88 \%)$. The maternal and neonatal demographics are shown in table 2; the cohort comprised 100 (19\%) primiparous and 499 (83\%) multiparous women. Of the multiparous women, 343 were known to be at risk because of a previous history of FNAIT. This group contained 50 siblings with ICH (15\%) and 23 cases of fetal demise (7\%), which can be classified as high-risk pregnancies.

Almost all pregnancies were singleton pregnancies (97\%). Regarding the 575 pregnancies with a known mode of delivery, 240 deliveries (39\%) were by vaginal route, including 4 assisted by ventouse or forceps. Three 
Table 3. Antenatal therapy

\begin{tabular}{lclll}
\hline & $\begin{array}{l}\text { Cases, } \\
\mathrm{n}(\%)\end{array}$ & $\begin{array}{l}\text { Mean PC at birth } \\
(\text { range }) \times 10^{9} / 1\end{array}$ & $\mathrm{n}$ ICH, IUFD, \\
& $\mathrm{n}$ & \\
\hline FBS & $21(3)$ & $131(5-302)$ & 0 & 1 \\
FBS + IVIG + steroids & $20(3)$ & $144(17-277)$ & 2 & 0 \\
FBS + IVIG & $75(12)$ & $166(12-391)$ & 2 & 0 \\
FBS + steroids & $8(1)$ & $71(4-166)$ & 1 & 0 \\
IVIG + steroids & $8(1)$ & $70(2-298)$ & 0 & 0 \\
IVIG & $138(22)$ & $122(4-354)$ & 4 & 1 \\
Steroids & $3(0.5)$ & $270^{\mathrm{a}}$ & 0 & 0 \\
No treatment & $338(55)$ & $86(1-405)$ & 14 & 2 \\
Unknown & 4 & & & \\
\hline
\end{tabular}

$\mathrm{PC}=$ Platelet count. ${ }^{\mathrm{a}} 1$ case.

hundred thirty-five (50\%) caesarean sections were performed, of which 289 were elective. Most deliveries were after 32 weeks of gestation (98\%). The mean birth weight was found to be $2,985 \mathrm{~g}$, with a mean gestational age of 37 weeks. The neonates were boys in $55 \%$ of the cases. When comparing boys and girls there was no significant difference in mean platelet counts at birth $\left(102 \times 10^{9} / 1\right.$ in both groups) or in mean birth weights (2,990 vs. $2,951 \mathrm{~g})$. Severe thrombocytopenia $\left(<50 \times 10^{9} / 1\right)$ was found in 235 cases (43\%), including 191 (31\%) neonates with platelet counts of less than $30 \times 10^{9} / 1$. Skin bleeding was reported in 94 cases (18\%); in most of these cases severe thrombocytopenia was found (94\%).

\section{Adverse Perinatal Outcomes}

In the database 4 cases of intrauterine fetal death (IUFD) and 3 neonatal deaths were found, giving an overall perinatal mortality of $1.14 \%$. In these cases no antenatal treatment had been given. Twenty-three neonates (4.5\%) were affected by ICH, 9 were first-born children. Of the other 14 cases, 4 had a sibling affected by ICH due to FNAIT.

HPA-1a was the concerning antigen in the majority of cases (83\%); in the other 4 cases, HPA-5a, HPA-5b, and a combination of HPA-1a/-5b was found. Nine of the neonates with ICH were treated during pregnancy; in 4 pregnancies treatment was started after ICH had been found. This is described in more detail in the next section.

A high proportion of the pregnancies complicated by ICH ended preterm: 12 of 23 children (52\%) were born before 37 weeks, and 2 children were born before 28 weeks of gestation. Two children suffering from ICH died in utero and 1 post term. The neonates with ICH were male in the majority (76\%) of cases. Furthermore 2 cases of intra-abdominal bleeding were found, 1 ending in IUFD at 24 weeks of gestation. In both pregnancies no antenatal treatment had been given.

\section{Antenatal Interventions}

In 273 pregnancies some form of antenatal treatment was given, ranging from cordocentesis with intrauterine platelet transfusion (IUPT) to maternal administration of intravenous immunoglobulin (IVIG), steroids, or a combination of those (table 3$)$. In most pregnancies $(n=138)$ a single treatment with IVIG was given, in 24 cases with 0.5 and in 102 cases with $1.0 \mathrm{~g} / \mathrm{kg} /$ week ( $\mathrm{n}=12$ unknown). In 124 pregnancies invasive treatment was offered. The two groups were comparable according the definition of high-risk FNAIT. There was no difference in the frequency of ICH or IUFD in previous siblings between the invasive and the non-invasive group [26/124 (21\%) vs. $28 / 138$ $(20 \%), \mathrm{p}=1.0]$.

The number of cordocenteses performed differed per centre. This ranged from $0 \%$ ( 0 per offered treatment in Scotland and Norway) to $100 \%$ (Canada). If we have a closer look at the major contributing centres in the database that performed cordocentesis during the study period (in the Netherlands and Sweden), a decline in invasive procedures is seen over the years (from 22\% in 2005 to $0 \%$ in 2008,2009 , and 2010). A single centre performed cordocentesis up to 2009 (in Canada; $\mathrm{n}=25$ ).

There were 9 cases of ICH reported in the antenatally treated group. Looking at the data in more detail, 5 cases of ICH occurred in the invasive procedure group; in 4 patients it seems likely that invasive therapy with fetal blood sampling (FBS) and serial IUPT (with IVIG or steroids) had been started before the diagnosis of ICH was made during ultrasound examination. In 1 case ICH was found, and subsequently invasive therapy was given.

The other 4 cases of ICH had been diagnosed before any antenatal treatment was given. In these pregnancies treatment with IVIG was started to prevent further worsening of bleeding. One of these pregnancies ended in IUFD 1 week after IVIG treatment had been started.

In the single FBS group, 1 IUFD was reported, seen 1 week after the first IUPT. It is unclear whether this was related to spontaneous fetal bleeding or to a complication of the procedure.

\section{Outcomes of High-Risk Pregnancies}

The clinical outcomes of the high-risk pregnancies $(n=73)$ are outlined in table 4 . Fifty-six cases received antenatal treatment (26 invasive and 30 non-invasive).
254

Fetal Diagn Ther 2017;41:251-257 DOI: $10.1159 / 000448753$
Kamphuis/Tiller/van den Akker/ Westgren/Tiblad/Oepkes 
Table 4. Clinical outcomes in the high-risk group

\begin{tabular}{lllll}
\hline & Cases, $\mathrm{n}(\%)$ & Median PC at birth $($ range $) \times 10^{9} / 1$ & ICH, $\mathrm{n}$ & IUFD, $\mathrm{n}$ \\
\hline Total high-risk cases & 73 & $96(1-391)$ & 3 & $1^{\mathrm{a}}$ \\
$\quad$ Platelet count $<50 \times 10^{9} / 1$ & $18(25)$ & & & \\
Platelet count $<30 \times 10^{9} / 1$ & $16(16)$ & & & \\
Antenatal treatment & 56 & $70(17-170)$ & 1 & \\
FBS + IVIG + steroids & 4 & $181(21-391)$ & & \\
FBS + IVIG & 21 & 253 & 2 & $1^{\mathrm{a}}$ \\
FBS & 1 & $60(2-296)$ & & \\
IVIG + steroids & 3 & $63(10-340)$ & & \\
IVIG & 27 & & & \\
\hline
\end{tabular}

${ }^{a} 1$ case suffering from ICH ended in IUFD.

In this high-risk group, 3 cases of ICH were found $(4.2 \%), 1$ resulting in IUFD. In 1 of these pregnancies antenatal treatment was given (IVIG + serial IUPT); in the other 2 cases IVIG was started after the diagnosis of $\mathrm{ICH}$.

\section{Discussion}

In this study we evaluated the treatment and outcome of more than 600 cases of FNAIT, the largest cohort of cases of FNAIT published on thus far. The majority of cases were collected after a first affected pregnancy. The overall frequency of ICH in our study group was 3.7\%, which is lower than previously reported [10]. This might well be explained by the several antenatal interventions performed in this group.

\section{Antenatal Treatment}

Different treatment regimens were offered to avoid recurrence of any burden, divided into invasive $(\mathrm{n}=124)$ and non-invasive $(\mathrm{n}=138)$. An important observation was that, although rare, all bleeding complications $(n=5)$ occurred in the invasively treated group (FBS + IUPT). No ICH cases were reported in the non-invasive group (maternal administration of IVIG). Although the exact cause of the adverse outcome in the invasively treated group could not be reliably assessed, at least we can conclude that there appears to be no benefit from invasive treatment over a non-invasive approach. Previous studies have calculated a cumulative risk of fetal loss per pregnancy of $6 \%$ directly related to complications of FBS and IUPT $[11,12]$.

Outcome of Pregnancies Complicated by FNAIT in a Large Cohort
Overall we can state that non-invasive antenatal treatment with maternal administration of IVIG appears successful in protecting fetuses and neonates from bleeding (138 cases treated, 0 cases of $\mathrm{ICH}$ reported). Furthermore, our study clearly indicates that over the years the invasive diagnostic and treatment approach in FNAIT has been almost completely replaced by safer non-invasive protocols.

Several reports have been published on IVIG treatment for FNAIT with a close to $100 \%$ success in preventing bleeding complications in fetuses and neonates [13-18]. Until now, the working mechanism of IVIG is not clear. Most likely, it acts on various levels, i.e. in maternal serum, at the level of placental transfer of $\operatorname{IgG}$, and in the fetal blood, blocking Fc receptors on macrophages [19]. Recent research by Yougbaré et al. [20] supports the hypothesis that IVIG may aid in protection against bleeding through a direct effect on endothelial cells, instead of merely causing a rise in platelets. They showed that impairment of angiogenesis rather than thrombocytopenia is the critical cause of ICH in FNAIT. In their murine model study, ICH only occurred in fetuses and neonates with anti- $\beta_{3}$ integrin-mediated - but not anti-GPIba-mediated - FNAIT, despite similar levels of thrombocytopenia in both groups. Only anti- $\beta_{3}$ integrin-mediated FNAIT reduced brain and retina vessel density, impaired angiogenic signalling, and increased endothelial cell apoptosis. This might be an explanation for the phenomenon of 'non-responders', i.e. fetuses not responding to IVIG with platelet counts remaining below $50 \times 10^{9} / 1$, reported to be around $20 \%[21]$. 
Outcomes in High-Risk Pregnancies

Most reports support the assumption that pregnant women with a previous child with intracranial bleeding compose the highest-risk group; the ICH recurrence rate in subsequent pregnancies is reported to be around 79\% [22]. Therefore, most clinicians caring for such pregnancies choose for a more aggressive approach as compared with the group in which the affected sibling did not have an ICH.

In our study, 73 FNAIT cases with a previous sibling with ICH or IUFD were reported; 56 of them received antenatal treatment, with 1 case ending in ICH and finally IUFD. In this pregnancy, invasive therapy was given (combination FBS + serial IUPT with IVIG). In this highrisk group 30 cases were treated completely non-invasively (all with IVIG, 3 in combination with steroids) and no ICH occurred.

\section{FNAIT and Fetal Gender}

In our large data set of pregnancies affected by FNAIT, $55 \%$ of the fetuses/neonates were male. Interestingly, in the group of children with $\mathrm{ICH}$, the proportion of males was much higher (76\%). A study by Tiller et al. [23] showed a clear association between the level of maternal HPA-1a antibodies and reduced birth weight in boys. How the sex of the offspring is involved in FNAIT remains to be explored.

The strengths of our study are its large sample size, which is 3 times larger than the previously largest series [24], the input of data by multiple international centres, and the variation in the interventions performed. The results obviously need to be interpreted with care, given the limitation that a large part of the data was collected retrospectively and a selection bias cannot be ruled out. More reliable data can only be obtained from prospective population screening studies.

\section{Conclusion}

Pregnancies affected by FNAIT, even with a severe history, now have an excellent prognosis. Our data confirm that there appears to be no benefit from invasive diagnostic or therapeutic procedures. Non-invasive management using IVIG with or without additional steroids prevents bleeding in the fetus or neonate in virtually all cases. Remaining issues for future research are the timing and optimal dose of IVIG, the role of gender, and the long-term neurologic outcome of the surviving affected children. In several countries, preparations are being made to implement population screening studies of FNAIT.

\section{Acknowledgements}

We thank all contributing centres (University Hospital Bristol, Leuven University Hospital, Queen Elizabeth University Hospital Glasgow, Helsinki University Hospital, Karolinska Institutet Stockholm, Uppsala University, Sheba Medical Centre, University Health Network Toronto, University College London, University Hospital North Norway, University Hospital South Norway, and Yale New Haven Hospital) for entering data into the NOICH registry database. We especially like to thank Prof. H.H.H. Kanhai and Prof. A. Husebekk for their contribution in setting up the NOICH registry database.

\section{Disclosure Statement}

None of the authors have any relevant financial, personal, political, intellectual, or religious interests to disclose. Funding was not obtained for this study.

\section{References}

1 Kaplan C: Platelet alloimmunity: the fetal/ neonatal alloimmune thrombocytopenia. Vox Sang 2002;83(suppl 1):289-291.

2 Warwick RM, Vaughan J, Murray N, Lubenko A, Roberts I: In vitro culture of colony forming unit-megakaryocyte (CFU-MK) in fetal alloimmune thrombocytopenia. $\mathrm{Br} \mathrm{J}$ Haematol 1994;88:874-877.

3 Spencer JA, Burrows RF: Feto-maternal alloimmune thrombocytopenia: a literature review and statistical analysis. Aust NZ J Obstet Gynecol 2001;41:45-55.

4 Mao C, Guo J, Chituwo BM: Intraventricular haemorrhage and its prognosis, prevention and treatment in term infants. J Trop Pediatr 1999;45:237-240.

5 Jocelyn LJ, Casiro OG: Neurodevelopmental outcome of term infants with intraventricular hemorrhage. Am J Dis Child 1992;146:194197.

6 Bonacossa IA, Jocelyn LJ: Alloimmune thrombocytopenia of the newborn: neurodevelopmental sequelae. Am J Perinatol 1996; 13:211-215.

7 Tiller H, Kamphuis MM, Flodmark O, Papadogiannakis N, David AL, Sainio S, Koskinen S, Javela K, Wikman AT, Kekomaki R, Kanhai HH, Oepkes D, Husebekk A, Westgren M: Fe- tal intracranial haemorrhages caused by fetal and neonatal alloimmune thrombocytopenia: an observational cohort study of 43 cases from an international multicentre registry. BMJ Open 2013;3:e002490.

8 Paridaans NP, Kamphuis MM, Taune Wikman A, Tiblad E, Van den Akker ES, Lopriore E, Challis D, Westgren M, Oepkes D: Low-dose versus standard-dose intravenous immunoglobulin to prevent fetal intracranial hemorrhage in fetal and neonatal alloimmune thrombocytopenia: a randomized trial. Fetal Diagn Ther 2015;38: 147-153. 
9 Kjeldsen-Kragh J, Killie MK, Tomter G, Golebiowska E, Randen I, Hauge R, Aune B, Øian P, Dahl LB, Pirhonen J, Lindeman R, Husby H, Haugen G, Grønn M, Skogen B, Husebekk A: A screening and intervention program aimed to reduce mortality and serious morbidity associated with severe neonatal alloimmune thrombocytopenia. Blood 2007;110: 833-839.

10 Kamphuis MM, Paridaans N, Porcelijn L, et al: Screening in pregnancy for fetal or neonatal alloimmune thrombocytopenia: systematic review. BJOG 2010;117:1335-1343.

11 Paidas MJ, Berkowitz RL, Lynch L, Lockwood CJ, Lapinski R, McFarland JG, Bussel JB: Alloimmune thrombocytopenia: fetal and neonatal losses related to cordocentesis. Am J Obstet Gynecol 1995;172:475-479.

12 Overton TG, Duncan KR, Jolly M, Letsky E, Fisk NM: Serial aggressive platelet transfusion for fetal alloimmune thrombocytopenia: platelet dynamics and perinatal outcome. Am J Obstet Gynecol 2002;186:826-831.

13 Birchall JE, Murphy MF, Kaplan C, Kroll H; European Fetomaternal Alloimmune Thrombocytopenia Study Group: European collaborative study of the antenatal management of feto-maternal alloimmune thrombocytopenia. Br J Haematol 2003;122:275-288.
14 Van den Akker ESA, Oepkes D, Lopriore E, Kanhai HHH: Noninvasive antenatal management of fetal and neonatal alloimmune thrombocytopenia: safe and effective. BJOG 2007;14:469-473.

15 Giers G, Wenzel F, Stockschläder M, Riethmacher R, Lorenz H, Tutschek B: Fetal alloimmune thrombocytopenia and maternal intravenous immunoglobulin infusion. Haematologica 2010;95:1921-1926.

16 Bertrand G, Drame M, Martageix C, Kaplan C: Prediction of the fetal status in noninvasive management of alloimmune thrombocytopenia. Blood 2011;117:3209-3213.

17 Yinon Y, Spira M, Solomon O, Weisz B, Chayen B, Schiff E, Lipitz S: Antenatal noninvasive treatment of patients at risk for alloimmune thrombocytopenia without a history of intracranial hemorrhage. Am J Obstet Gynecol 2006;195:1153-1157.

18 Berkowitz RL, Kolb EA, McFarland JG, Wissert M, Primani A, Lesser M, Bussel JB: Parallel randomized trials of risk-based therapy for fetal alloimmune thrombocytopenia. Obstet Gynecol 2006;107:91-96.

19 Ni H, Chen P, Spring CM, Sayeh E, Semple JW, Lazarus AH, Hynes RO, Freedman J: A novel murine model of fetal and neonatal alloimmune thrombocytopenia: response to intravenous IgG therapy. Blood 2006;107: 2976-2983.
20 Yougbaré I, Lang S, Yang H, Chen P, Zhao X, Tai WS, Zdravic D, Vadasz B, Li C, Piran S, Marshall A, Zhu G, Tiller H, Killie MK, Boyd S, Leong-Poi H, Wen XY, Skogen B, Adamson SL, Freedman J, Ni H: Maternal anti-platelet $\beta 3$ integrins impair angiogenesis and cause intracranial hemorrhage. J Clin Invest 2015; 125:1545-1556

21 Tiller H, Husebekk A, Skogen B, KjeldsenKragh J, Kjaer M: True risk of fetal/neonatal alloimmune thrombocytopenia in subsequent pregnancies: a prospective observational follow-up study. BJOG 2016;123:738744.

22 Radder CM, Brand A, Kanhai HH: Will it ever be possible to balance the risk of intracranial haemorrhage in fetal or neonatal alloimmune thrombocytopenia against the risk of treatment strategies to prevent it? Vox Sang 2003; 84:318-325.

23 Tiller H, Killie MK, Husebekk A, Skogen B, Ni H, Kjeldsen-Kragh J, Øian P: Platelet antibodies and fetal growth: maternal antibodies against fetal platelet antigen 1a are strongly associated with reduced birth weight in boys. Acta Obstet Gynecol Scand 2012;91:79-86.

24 Ghevaert C, Campbell K, Walton J, Smith GA, Allen D, Williamson LM, Ouwehand WH, Ranasinghe E: Management and outcome of 200 cases of fetomaternal alloimmune thrombocytopenia. Transfusion 2007;47:901-910. 